\title{
Performance evaluation / Évaluation de performances
}

Revue française d'automatique, d'informatique et de recherche opérationnelle. Recherche opérationnelle, tome 32, $\mathrm{n}^{\circ} 3$ (1998), p. 2-3 (pages préliminaires).

<http://www.numdam.org/item?id=RO_1998_32_3_2_0>

(C) AFCET, 1998, tous droits réservés.

L'accès aux archives de la revue « Revue française d'automatique, d'informatique et de recherche opérationnelle. Recherche opérationnelle » implique l'accord avec les conditions générales d'utilisation (http://www.numdam.org/ legal.php). Toute utilisation commerciale ou impression systématique est constitutive d'une infraction pénale. Toute copie ou impression de ce fichier doit contenir la présente mention de copyright.

\section{Numdam}

Article numérisé dans le cadre du programme

Numérisation de documents anciens mathématiques

http://www.numdam.org/ 


\section{Performance Evaluation/Evaluation de Performances}

e numéro spécial fait suite au Sixième Atelier d'Évaluation de Performances organisé par le Laboratoire PRiSM de l'Université de Versailles-Saint-Quentin les 25-28 novembre 1996 sous le parrainage du PRC-GdR PRS (Parallélisme, Réseaux et Systèmes).

Le domaine de l'évaluation de performances porte sur la modélisation et l'analyse quantitative de systèmes à événements discrets. Les systèmes à événements discrets constituent une classe très importante de systèmes puisqu'ils se rencontrent dans de nombreux domaines dont on peut citer comme exemples: les systèmes informatiques, les réseaux de télécommunication et les systèmes de production industriels. Tous ces systèmes ont des comportements très complexes et il est donc nécessaire de disposer de méthodes et d'outils d'évaluation de performances adaptés à la classe des systèmes à événements discrets et qui sont indispensables pour aider à optimiser ces systèmes lors de leur conception et de leur exploitation, en intégrant éventuellement des problèmes de fiabilité.

La modélisation de systèmes à événements discrets peut être abordée avec l'aide de divers outils de modélisation, dont les principaux sont les réseaux de files d'attente, les réseaux de Petri et les réseaux d'automates. La plupart des modèles développés pour analyser le comportement de systèmes tels que ceux cités précédemment sont de nature stochastique, c'est-à-dire qu'une partie du comportement du modèle est probabiliste. Leur analyse repose sur des outils théoriques adaptés à l'étude de modèles stochastiques : les chaînes de Markov mais aussi les processus ponctuels et 1 ' « algèbre » $(\max ,+)$. Les méthodes de résolution reposent sur différentes techniques : méthodes analytiques, méthodes numériques, simulation, techniques d'approximation.

La communauté française du domaine de l'évaluation de performance est très dynamique et a acquis une reconnaissance importante au niveau international, que ce soit pour ses travaux à caractère théorique ou dans le cadre d'études appliquées. Ce numéro spécial contient un ensemble d'articles sélectionnés parmi ceux présentés à l'Atelier d'Évaluation de Performances de Versailles, qui a réuni une grande partie de la communauté française. Certains sont à caractère théorique ou méthodologique ; d'autres s'intéressant plus à des problématiques appliquées.

L'article de G. Hébuterne présente une réflexion sur les modèles de files d'attente à temps discret. De tels modèles se rencontrent fréquemment dans 
les systèmes informatiques et les réseaux de télécommunication, en particulier les réseaux à haut débit de type ATM. L'auteur met en évidence, au moyen d'un certain nombre d'exemples, le caractère spécifique du temps discret dans le comportement des modèles.

L'article de H. Kaddeche, A. L. Beylot et M. Becker porte sur l'évaluation de performances d'un serveur multimédia multi-disque délivrant des séquences vidéo à des utilisateurs via un réseau à haut débit. Le système est modélisé par un réseau de files d'attente dont les performances (temps de réponse et charge maximale) sont obtenues par simulation.

L'article de C. Labbé, F. Reblewski et J. M. Vincent propose une approche originale pour l'évaluation de performances de systèmes modélisés par des réseaux de files d'attente à temps discret, par exemple des réseaux ATM. Cette approche consiste à émuler le réseau à l'aide d'une architecture reconfigurable très rapide qui conduit à des temps de calcul particulièrement faibles comparés à ceux de l'approche classique par simulation.

L'article de S. Bulteau et M. El Khadiri porte sur l'évaluation de la fiabilité d'un réseau de transport stochastique où les capacités des arcs sont des variables aléatoires. L'auteur présente une approche utilisant une méthode de décomposition de l'espace d'états pour mettre en œuvre une technique de simulation de type Monte Carlo.

L'article de S. Haddad, P. Moreaux et G. Chiola présente une nouvelle méthode d'analyse de réseaux de Petri stochastiques dans lesquels les temps de franchissement des transitions sont caractérisés par des lois de Cox. Cette méthode efficace repose sur l'utilisation conjointe d'une analyse qualitative du réseau et de la technique de décomposition tensorielle.

L'article de P. Fernandes, B. Plateau et W. J. Stewart propos une nouvelle technique numérique pour calculer la solution stationnaire de la chaîne de Markov associée à un réseau d'automate stochastique. La contribution de cet article est de rendre plus efficace la méthode de décomposition tensorielle en la couplant avec des techniques d'analyse numérique comme le préconditionnement et la méthode GMRES.

L'article de J. M. Fourneau, N. Pekergin et D. Verchère s'intéresse à une classe de réseaux de files d'attente appelée G-réseaux avec synchronisations cycliques. Ces réseaux sont une généralisation des réseaux de files d'attente classiques permettant de modéliser certains phénomènes de synchronisation au moyen de signaux. Les auteurs montrent que sous certaines hypothèses, de tels réseaux de files d'atttente possèdent encore une solution à forme-produit. 